ARTÍCULO DE REVISIÓN

\title{
EL CIGARRILLO ELECTRÓNICO: UN PROBLEMA DE SALUD PÚBLICA EMERGENTE
}

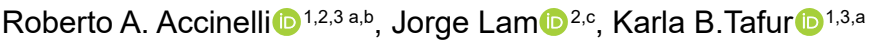 \\ 1 Hospital Cayetano Heredia, Lima, Perú. \\ 2 Instituto de Investigaciones de la Altura, Universidad Peruana Cayetano Heredia, Lima, Perú. \\ 3 Facultad de Medicina, Universidad Peruana Cayetano Heredia, Lima, Perú. \\ ${ }^{\mathrm{a}}$ Médico neumólogo; ${ }^{\mathrm{b}}$ magíster en Salud Pública; ${ }^{\mathrm{c}}$ estudiante de medicina humana
}

\section{RESUMEN}

Desde el 2004 se dispone del cigarrillo electrónico (CE), dispositivo que calienta nicotina y la administra formando parte de un vapor. Presentamos una revisión narrativa del CE y su efecto en la salud. Su uso es para dejar de fumar, en lo que la evidencia es baja, terminando usándolo a la vez que el cigarrillo que no pudieron dejar. Además, los que nunca fumaron, principalmente adolescentes y jóvenes, se inician en su consumo. Su uso eleva en el aire los niveles de nicotina, partículas, compuestos orgánicos volátiles, hidrocarburos aromáticos policíclicos, carbonilos y metales como aluminio. In vitro el CE causa inflamación, estrés oxidativo y es tóxico para múltiples tipos de células, incluyendo células pulmonares, endoteliales y células madre. Produce incremento de la susceptibilidad a infecciones virales y bacterianas. Comparado con cigarrillos, el CE produce un número mayor y más intenso de genes suprimidos. A la broncoscopia las vías aéreas están friables y eritematosas, y el epitelio bronquial con expresión diferenciada de proteínas. Se le ha asociado con tos, síntomas de bronquitis y con la epidemia de insuficiencia respiratoria por neumonitis que ha llevado a varias decenas de personas a la muerte. Por sus efectos dañinos el CE debería solo usarse por prescripción médica, como una medida para ayudar a dejar el tabaco, y estar prohibido su uso en interiores y ambientes públicos. Como no se han determinado los componentes del CE responsables de las muertes asociadas, su uso debería ser proscrito hasta que se conozcan cuáles son estos factores.

Palabras clave: Cigarrillo Electrónico; Nicotina; Saborizantes; Neumonitis (fuente: DeCS BIREME).

\section{THE ELECTRONIC CIGARETTE: AN EMERGING PUBLIC HEALTH PROBLEM}

\begin{abstract}
Since 2004, the electronic cigarette (EC) is available, a device that heats nicotine and administers it as part of a vapor. We present a narrative review of the $\mathrm{EC}$ and its effect on health. Its use is to stop smoking, in which the evidence is low, and ends up being used at the same time as the cigarette they could not quit. In addition, those who never smoked, mainly teenagers and young people, begin consumption. Its use raises the levels of nicotine, particles, volatile organic compounds, polycyclic aromatic hydrocarbons, carbonyls and metals such as aluminum. In vitro, the EC causes inflammation, oxidative stress and is toxic to multiple cell types, including lung, endothelial and stem cells. Produces increased susceptibility to viral and bacterial infections. Compared to cigarettes, the EC produces a larger and more intense number of deleted genes. At bronchoscopy the airways are friable and erythematous, and the bronchial epithelium with a differentiated protein expression. It has been associated with cough, bronchitis symptoms and the respiratory failure by pneumonitis epidemic that has led several dozen people to death. Because of its harmful effects, the EC should only be used by medical prescription, as a measure to help quit tobacco, and its use in indoor and public environments be prohibited. As the EC components responsible for the associated deaths have not been determined, their use should be banned until these factors are known.
\end{abstract}

Tafur K. El cigarrillo electrónico: un problema de salud pública emergente. Rev Peru Med Exp Salud Publica. 2020;37(1):122-28. Doi: https://doi org/10.17843/rpmesp.2020.371.4780

Correspondencia: Roberto A. Accinelli; roberto.accinelli@upch.pe

Recibido: 06/09/2019 Aprobado: 29/01/2020 En línea: 23/03/2020

\section{INTRODUCCIÓN}

En 1954 Doll y Hill encontraron que los grandes fumadores tenían 40 veces más riesgo de fallecer por cáncer de pulmón ${ }^{(1)}$. Así quedó fehacientemente demostrado el efecto dañino del tabaco en la salud. Casi la mitad de las muertes en 12 tipos diferentes de cáncer son atribuibles al consumo de tabaco ${ }^{(2)}$. El tabaquismo también está relacionado con enfermedad pulmonar 
obstructiva crónica (EPOC), enfermedad coronaria, accidente cerebrovascular (ACV), asma, infertilidad en mujeres, embarazo ectópico, prematuridad y/o bebés con un bajo peso al nacer, diabetes mellitus tipo 2, ceguera, cataratas y degeneración macular relacionada con la edad ${ }^{(3)}$. Más de 7 millones de personas mueren al año a causa del tabaco, de las cuales más de 6 millones son fumadores y alrededor de 890000 personas están expuestas al humo ajeno. El humo del tabaco contiene más de 4000 productos químicos, de los cuales al menos 250 son nocivos y más de 50 cancerígenos. Se han implementado políticas de control con las cuales se ha logrado disminuir la prevalencia de consumo de tabaco en los varones mayores de 15 años de $41,2 \%$ en 1980 a 31,1\% en el 2012, y en las mujeres de $10,6 \%$ a $6,2 \%$. Sin embargo, debido al crecimiento de la población el número total de fumadores ha aumentado de forma neta en casi 250 millones ${ }^{(4)}$. En los países desarrollados la disminución ha sido más notable, mientras que los países con ingresos bajos o medios concentran casi el $80 \%$ de los más de mil millones de fumadores.

Más de la mitad de los fumadores desean abandonar el consumo de tabaco, pero gracias al efecto adictivo de la nicotina, solo $2 \%$ a $3 \%$ lo logra cada año ${ }^{(5)}$. En el 2003 Hon Lik patentó un sistema electrónico de entrega de nicotina, también llamado cigarrillo electrónico (CE), con la finalidad de eliminar los efectos nocivos de los otros productos presentes en el cigarrillo. El CE fue introducido al mercado al año siguiente como otro elemento más de ayuda para dejar de fumar ${ }^{(6)}$. Diez años después sus ventas habían alcanzado los $2,5 \times 10^{6}$ millones de dólares ${ }^{(7)}$ y se espera que sobrepasen la de los cigarrillos convencionales para el $2023^{(8)}$.

En los Estados Unidos (EE. UU.), la prevalencia del consumo de tabaco ha disminuido desde el 2005 hasta el 2016 en los adultos mayores de 18 años, además, la proporción de fumadores que han logrado abandonar su consumo ha aumentado. Sin embargo, la prevalencia del consumo del CE se ha duplicado entre los años 2013 y 2014 entre los adultos de 18 a 24 años, y entre los escolares el aumento ha sido del $1,5 \%$ al $16 \%$ entre 2011 y $2015^{(9)}$. En otros países, el uso del CE también es elevado entre los jóvenes, por ejemplo, Polonia reporta una tasa de $62,1 \%$ y Canadá $71,9 \%{ }^{(10)}$.

En el Perú, la legislación contra el consumo de tabaco ha permitido que su prevalencia haya disminuido de $44,5 \%$ en 1998 a $21,1 \%$ en el $2010^{(11)}$. Entre los universitarios peruanos el consumo de tabaco en los últimos 30 días disminuyó desde el 2012 al 2016 de 22,6\% a 16\%. Esta disminución es incluso mayor entre los estudiantes menores de 18 años, sin embargo, este grupo es quien más utiliza los CE (16,3\%) en comparación con los mayores de 25 años (8,8\%). La prevalencia de uso alguna vez en la vida de CE entre los universitarios peruanos es de $12,6 \%{ }^{(12)}$.

En el presente artículo revisaremos todo lo concerniente al CE y su efecto en la salud humana. Hemos realizado una revisión narrativa desde el 1 de diciembre del 2018 al
18 de noviembre del 2019. Se realizó la búsqueda en la base de PubMed empleando los términos e-cigarettes y electronic cigarettes. Se seleccionaron aquellos artículos que contenían información relacionada con cesación de tabaquismo, efectos en el ambiente, efectos secundarios en los seres vivos, insuficiencia respiratoria y reglamentación sobre su uso.

\section{EL CIGARRILLO ELECTRÓNICO}

Es un dispositivo que libera vapores de nicotina y saborizantes por calentamiento electrónico de un líquido que utiliza una base de propilenglicol/glicerina vegetal (PG/VG) y no por combustión. Los $\mathrm{CE}$ consisten en un tubo de plástico o de acero inoxidable, un serpentín de calentamiento electrónico, un cartucho de líquido con PG/GV como estabilizante, cantidades variables de nicotina, aditivos aromatizantes y saborizantes, una batería de litio y una cámara de atomización. La temperatura en el centro de la bobina de calentamiento alcanza más de $350^{\circ} \mathrm{C}$. Cuando el usuario activa al CE, el calentador atomiza el líquido, lo que produce un aerosol, conocido como vapor, con una fase particulada y otra gaseosa, cuya composición química difiere de la del líquido del cartucho ${ }^{(6,13)}$. De allí que al acto de fumar CE se le llame vapear. Según la marca, el contenido de nicotina puede variar desde 0 a $36 \mathrm{mg} / \mathrm{mL}^{(14)} \mathrm{y}$ también pueden ser totalmente diferentes las baterías, los elementos de calentamiento y los saborizantes en el líquido del cartucho. Hay alrededor de 7700 sabores diferentes en venta, como sabor a tabaco, chocolate, menta, fruta, café, tetrahidrocannabinol (THC), entre otros. Hoy en día se pueden personalizar incluso a pedido del consumidor ${ }^{(15)}$.

Existen cuatro generaciones de CE que han cambiado en diseño para permitir al usuario un mayor control sobre la concentración de nicotina, la composición del líquido y la forma en que lo vaporiza. El último de estos es conocido como JUUL un CE plano y rectangular como una memoria externa y recargable en un puerto USB que usa sales de nicotina en lugar de su base libre empleada en los primeros $\mathrm{CE}$, que es alcalina $^{(16-18)}$. Inicialmente se prefirió su base libre porque el pH elevado aumenta la absorción de nicotina por el sistema respiratorio ${ }^{(19)}$, con lo que habría más ventas de $\mathrm{CE}$, esto a semejanza de los cigarrillos convencionales, cuya demanda es mayor en las marcas con el rango más alto de nicotina extraíble ${ }^{(20)}$. Pero al ser la base libre amarga causa un golpe en la garganta que genera tos. Esto ha querido evitarse con el JUUL, tan pequeño que puede ser usado incluso durante clases sin que los profesores lo adviertan, pues no causa tos ${ }^{(21)}$. Sin embargo, para lograr adicción a su uso los $0,7 \mathrm{~mL}$ de su cámara llevan la solución de sales de nicotina al $5 \%(59 \mathrm{mg} / \mathrm{mL})^{(22)}$. Antes de junio de 2015, año en que aparece el JUUL, los CE llevaban nicotina al $1-2 \%{ }^{(23)}$.

En un estudio se encontraron 71 proveedores de líquido para CE a granel (frascos con más de $30 \mathrm{~mL}$ ) con concentraciones de sales de nicotina mayores del $5 \%$, equivalente a más 
de 40 paquetes de cigarrillos, con múltiples sabores dulces y frutados ${ }^{(24)}$. Esta situación aumenta la posibilidad de intoxicación involuntaria al ingerir el líquido del cartucho, sobre todo en los niños.

\section{EL CIGARRILLO ELECTRÓNICO Y SU UTILIDAD PARA DEJAR DE FUMAR}

La revisión sistemática de Vanderkam y col. demostró que, a los tres meses de usar $\mathrm{CE}$, los fumadores consumían menos cigarrillos convencionales (RR 2,55), pero a los seis meses el porcentaje que se mantenía sin fumar disminuía (RR 1,30) ${ }^{(25)}$. En la revisión sistemática de Liu y col., una de las más grandes realizada con 35665 participantes de 14 publicaciones, se halló una reducción del consumo de tabaco del $48,3 \%$ al 58,7\% y de cesación del tabaquismo del $13,2 \%$ al $22,9 \%{ }^{(26)}$. En la cohorte COPDGene, $91 \%$ de los usuarios de CE utilizaban estos dispositivos con la intención de disminuir su consumo de tabaco, pero solo el $47 \%$ redujo el número de cigarrillos convencionales. La cohorte no evidenció una reducción en el consumo de tabaco o un efecto en la progresión de la EPOC ${ }^{(27)}$. En otra revisión de cuatro estudios de eficacia y 22 artículos de seguridad, se encontró que los CE lograban una mayor prevalencia puntual de abstinencia tabáquica al mes (RR 1,71) que no se mantuvo a los tres ni seis meses de seguimiento ${ }^{(28)}$. En los EE. UU., el $40 \%$ de los consumidores de tabaco usan al menos dos tipos de productos, siendo la combinación más común cigarrillos convencionales más $C E^{(29)}$. En el Reino Unido ha aumentado el uso de CE entre los fumadores regulares del 2,7\% en 2010 a 6,7\% en 2012, porque las personas que intentan dejar de fumar siguen haciéndolo y además usan el $\mathrm{CE}^{(30)}$.

\section{EFECTOS ADVERSOS DEL CIGARRILLO ELECTRÓNICO}

Los eventos adversos en once estudios con 16406 personas fluctuaron del $49,1 \%$ al 51,6\%, siendo los más frecuentes la irritación de la boca o garganta, ansiedad, estado de ánimo depresivo, náuseas e insomnio ${ }^{(25)}$. Los eventos adversos disminuyen con el tiempo, pero fueron más frecuentes que con los de parches de nicotina (19,7\% versus $11,8 \%$, RR 1,97, IC95\% 1,05 - 3,68) ${ }^{(28)}$. En los EE. UU. el 2015 hubo aproximadamente 3000 exposiciones consecuencia del contenido del líquido de los CE, la mayoría en niños menores de cinco años, lo que derivó en mil atenciones médicas ${ }^{(31)}$.

\section{EFECTO DEL CIGARRILLO ELECTRÓNICO EN AMBIENTES CERRADOS}

Los cigarrillos electrónicos emiten numerosos productos químicos que afectan negativamente la calidad del aire interior, por ejemplo, incrementan los niveles de nicotina, partículas, hidrocarburos aromáticos policíclicos y aluminio en el aire. Con la máquina de fumar Palaczbot (Technical University of Lodz) ${ }^{(32)}$ y en los vapeadores los valores de $\mathrm{PM}_{2,5}{ }^{(33)}$ sobrepasan el límite recomendado por la OMS, siendo más altos cuando en el CE se usan líquidos que contienen nicotina ${ }^{(16)}$.

Las emisiones de nicotina ambiental de los CE difieren entre las marcas, superponiéndose sus valores a los encontrados en el del humo del cigarrillo convencional ${ }^{\left({ }^{34}\right)}$. En el líquido de los cartuchos hay compuestos carbonílicos, como aldehído-formaldehido (FA) y acetaldehído (AA), reportados como factores de riesgo de cáncer ${ }^{(35)}$.

Por tanto, los CE no están libres de emisiones que afectan la calidad del aire y su uso puede exponer involuntariamente a los que no vapean a la nicotina y a los productos tóxicos que se liberan en el vapor.

\section{EFECTO DEL CIGARRILLO ELECTRÓNICO EN LOS SERES VIVOS}

\section{Síntomas y enfermedades respiratorias}

En los usuarios de CE hay una mayor prevalencia de síntomas respiratorios independientemente de si hay consumo de tabaco concomitante o no. Una sola sesión de vapeo con CE induce una inhibición significativa de la sensibilidad al reflejo de la tos. Habría una acción periférica y un efecto antitusivo, probablemente central, demostrable 15 minutos después de la exposición al $\mathrm{CE}^{(36)}$. Tan solo cinco minutos de exposición al CE causó estrés oxidativo e incremento de la resistencia dinámica de la vía aérea en relación con la disminución de la fracción exhalada de óxido nítrico (FeNO) ${ }^{(37)}$. La exposición durante un minuto al propilenglicol produjo una reducción del $\mathrm{VEF}_{1} / \mathrm{CVF}^{(38)}$.

Las tasas de síntomas bronquíticos crónicos aumentan en adolescentes usuarios de $\mathrm{CE}^{(39)}$. Usar $\mathrm{CE}$ se asocia con asma en adolescentes, tanto con asma actual como con el antecedente ${ }^{(40)}$. La prevalencia de uso de CE fue mayor entre los que tuvieron un ataque de asma y los que faltaron más días a la escuela por síntomas graves ${ }^{(41)}$. No hay estudios a largo plazo con el CE, pero el tener asma en la adolescencia se asocia 50 años después con un mayor riesgo de EPOC, una función ventilatoria reducida y una esperanza de vida más corta ${ }^{(42)}$.

Exfumadores seguidos por tres meses mostraron una mejoría de los síntomas nasales (SNOT-22) y del aclaramiento mucociliar (CCM), pero en aquellos que usaron $\mathrm{CE}$ como terapia para lograrlo no varió el CCM, pero sí los síntomas, mejorando más tanto CCM como SNOT-22 en quienes dejaron de fumar $\sin \mathrm{CE}^{(43)}$.

\section{Células}

Neutrófilos y macrófagos de individuos sanos, no fumadores, expuestos al extracto del aerosol del CE incrementaron la metaloproteinasa de matriz, MMP-9, y la quimiocina, 
CXCL8, similar a lo que sucede con el uso de cigarrillos convencionales ${ }^{(44)}$.

La exposición de células del epitelio respiratorio al aerosol de los CE produce alteración en el transporte de iones a nivel de estas células, por inhibición de la función del regulador de la conductancia transmembrana de la fibrosis quística (CFTR) inducida por la acroleína ${ }^{(45)}$.

Algunos de los saborizantes en el líquido de los CE afectaron en forma dosis dependiente la señalización por calcio y disminuyeron la viabilidad y proliferación de células del epitelio respiratorio humano ${ }^{(46)}$.

En un modelo tisular, cinco de los siete aromatizantes analizados provocaron la muerte de las células epiteliales traqueales. Se encontró alteraciones en la fisiología celular por la vainillina y el saborizante de chocolate 2,5-dimetilpirazina, que produce una activación dependiente de la proteína quinasa $\mathrm{A}$ (PKA) del CFTR ${ }^{(47)}$. Este mal funcionamiento del CFTR se ha asociado con asma y EPOC ${ }^{(48)}$.

La base PG/VG sola en aerosol aumentó la proteína MUC5AC tanto en los cultivos epiteliales de las vías respiratorias humanas como en los epitelios nasales murinos. Los líquidos del CE entraron rápidamente en las células y la base PG/VG redujo la fluidez de la membrana y afectó la difusión de proteínas ${ }^{(49)}$.

\section{Animales}

Los niveles de cotinina sérica en ratones expuestos al aerosol de CE son comparables a los niveles de este metabolito en humanos fumadores y ratones expuestos al humo de cigarrillos convencionales. Esta exposición indujo producción de mucina, fibrosis de la vía aérea, expresión de proteasas, hiperreactividad bronquial y destrucción del tejido pulmonar. La exposición solo a glicerina (vehículo del líquido) indujo efectos menores ${ }^{(50)}$.

Ratones expuestos al aerosol del CE presentaron alteraciones de la capacidad de aclaramiento bacteriano pulmonar, tras la infección intranasal con Streptococcus pneumoniae. Este defecto en el aclaramiento es debido en parte a alteraciones en la fagocitosis de macrófagos alveolares. En respuesta a la infección con el virus de la influenza $\mathrm{A}$, los ratones expuestos presentaron títulos más altos de virus a nivel pulmonar, así como mayor mortalidad ${ }^{(51)}$.

\section{Inmunidad}

El líquido del CE sin nicotina causa producción de IL-6 e infección por el rinovirus humano, que se amplifica cuando se añade nicotina y se bloquea con el clon 1 del epitelio nasal (SPLUNC1). Ratones deficientes en SPLUNC1 aumentan la carga de rinovirus en el pulmón ${ }^{(52)}$.

El CE incrementa la expresión nasal de factor receptor activador de plaquetas (PAFR), y la adherencia in vitro a células de la vía aérea que se atenuó cuando se usó CV3988 un bloqueador de PAFR. Su vapor aumenta la expresión del PAFR en la mucosa nasal de ratones y la colonización nasofaríngea por neumococo. La adherencia se disminuyó con acetilcisteína ${ }^{(53) .}$

La exposición al aerosol de CE aumenta la virulencia de Staphylococcus aureus resistente a la meticilina, ya que se altera su carga superficial y la formación de la biopelícula, lo que le confiere mayor resistencia a la destrucción por péptidos antimicrobianos y macrófagos. Además, la capacidad de las células epiteliales humanas para destruir patógenos disminuía ${ }^{(54)}$.

La exposición de monocitos de sangre periférica y de macrófagos alveolares extracto de $\mathrm{CE}$ redujo significativamente la producción de TNF- $\alpha$, IFN- $\gamma$ e IL-10, mientras que con solo nicotina disminuyó IL-10 y TNF- $\alpha$, citoquinas claves en la respuesta inmune contra las micobacterias. Después de cinco días en cultivo y con además con exposición a extracto de $\mathrm{CE}$, estas células tenían cargas bacilares intracelulares significativamente más altas, señal del impacto nocivo en la capacidad de los macrófagos humanos para contener el crecimiento de micobacterias ${ }^{(55)}$.

\section{Genética}

A los usuarios de CE se les ha encontrado mediante broncoscopía vías respiratorias friables y eritematosas. $\mathrm{Y}$ al estudiar las células epiteliales de las vías respiratorias se halló que unas 300 proteínas se expresaban de manera diferente, 78 proteínas tuvieron alteraciones entre los fumadores de cigarrillos, mientras que 113 estaban alteradas entre aquellos que solo usaban $\mathrm{CE}^{(49)}$.

Los líquidos del CE redujeron la viabilidad celular y causaron fragmentación del ADN en cultivos de tejido faríngeo humano, siendo mayor el daño cuando tenían saborizantes a frutas ${ }^{(56)}$. Varios sabores en el extracto de vapor de los CE demostraron ser citotóxicos para el epitelio de las vías respiratorias, existen variaciones entre las diferentes marcas y líneas celulares ${ }^{(57)}$.

La exposición al aerosol de CE induce estrés oxidativo y alteraciones moleculares en el epitelio respiratorio. La nicotina y los saborizantes del líquido de los cartuchos tienen un efecto sinérgico en la inducción de genes de estrés oxidativo ${ }^{(58)}$.

\section{Daño pulmonar asociado al uso de CE (EVALI)}

EVALI el acrónimo en inglés (E-cigarette or vaping associated lung injury) del vapeo. Es una enfermedad con una lesión pulmonar grave y síntomas constitucionales y gastrointestinales ${ }^{(59)}$. En el 2012 se reportó el primer caso donde mediante lavado broncoalveolar se hallaron numerosos macrófagos cargados de grasa, por lo que se hizo el diagnóstico de neumonía lipoidea ${ }^{\left({ }^{60}\right)}$. Hasta marzo del 2018 habían sido reportados nueve casos más, uno de los diez casos falleció por daño alveolar difuso ${ }^{(61)}$. 
En julio de este año, el Centers for Disease Control and Prevention (CDC) reportó un número inusual de personas internadas por enfermedad respiratoria que tenían todas como antecedente común usar CE. Hasta el cinco de noviembre de 2019, 2051 casos de EVALI han sido reportados al CDC en 49 de los 50 estados de los EE. UU. habiendo fallecido 39 (1,9\%). El 70\% eran varones con una edad promedio de 24 años, el 79\% era menor de 35 años. El 86\% informó que usaba $\mathrm{CE}$ con THC y en $34 \%$ de uso exclusivo, mientras que el $64 \%$ nicotina y en $11 \%$ de uso exclusivo. Cuando se estudió en 29 muestras el contenido del lavado broncoalveolar, se halló en el $100 \%$ acetato de vitamina E, que se usa como aditivo en el CE, en el $82 \%$ se identificó THC y nicotina en el $62 \%{ }^{(62)}$.

Radiológicamente los casos demuestran una colección heterogénea de patrones de neumonitis que incluyen neumonía eosinofílica aguda, neumonía organizada, neumonía lipoidea, daño alveolar difuso y síndrome de dificultad respiratoria aguda (SDRA), hemorragia alveolar difusa, neumonitis por hipersensibilidad, y la rara neumonitis intersticial de células gigantes ${ }^{(63,64)}$. Pero cuando los pacientes tuvieron biopsias pulmonares, los hallazgos en 17 pacientes fueron de lesión pulmonar aguda, incluyendo neumonitis fibrinosa aguda, daño alveolar difuso o neumonía organizada, generalmente bronquiolocéntrica y acompañada de bronquiolitis ${ }^{(65)}$. Hallazgos semejantes se han encontrado en las biopsias de otros ocho $\operatorname{casos}^{(66)}$.

\section{REGLAMENTACIÓN SOBRE EL USO DEL CIGARRILLO ELECTRÓNICO}

Hay 98 países que tienen leyes nacionales/federales que regulan el uso de los $\mathrm{CE}^{(67)}$ : su venta esta prohibida en 25 países y requiere autorización de mercado en otros 17. En los países que permiten la venta de cigarrillos electrónicos, la edad mínima de compra es de 18 años en 23 países, de 19 en la República de Corea y de 21 en Honduras ${ }^{(68)}$.

\section{REFERENCIAS BIBLIOGRÁFICAS}

1. Doll R, Hill AB. The mortality of doctors in relation to their smoking habits. BMJ. 1954;1(4877):1451-1455. doi:10.1136/bmj.1.4877.1451

2. Siegel RL, Jacobs EJ, Newton CC, Feskanich D, Freedman ND, Prentice $\mathrm{RL}$, et al. Deaths due to cigarette smoking for 12 smoking-related cancers in the United States. JAMA Intern Med. 2015;175(9):1574-1576. doi:10.1001/jamainternmed.2015.2398

3. National Center for Chronic Disease Prevention and Health Promotion (US) Office on Smoking and Health. The health consequences of smoking - 50 years of progress: a report of the surgeon general. Atlanta (GA): Centers for Disease Control and Prevention (US); 2014. [acceso el 18 de noviembre del 2019]. Disponible en: https://www.ncbi.nlm. nih.gov/books/NBK179276/

4. Ng M, Freeman M, Fleming T, Robinson M, Dwyer-Lindgren L, Thomson $\mathrm{B}$, et al. Smoking prevalence and cigarette consumption in 187 countries, 1980 - 2012. JAMA. 2014; 311:183-192. doi:10.1001/jama.2013.284692

5. Aveyard P, West R. Managing smoking cessation. BMJ.2007;335(7609):3741. DOI: $10.1136 / \mathrm{bmj} .39252 .591806 .47$
Los CE entraron al mercado sin pruebas exhaustivas de toxicología preclínica o ensayos de seguridad a largo plazo indispensables para introducir productos terapéuticos o médicos convencionales. Las compañías tabacaleras buscan atenuar los riesgos de los CE y destacarlos como una «alternativa mucho más segura» a la vez que promueven sabores atractivos para los niños. Con la aparición de la epidemia de muertes asociadas al uso de CE, el CDC recomienda no usar CE que contengan THC y que el acetato de vitamina $\mathrm{E}$ ni ninguna otra sustancia no indicada por el fabricante se agregue al CE. Con los severos efectos negativos del CE, que incluyen hasta la muerte de quienes los usan, descritas ya en América Latina ${ }^{(69)}$, debería el gobierno peruano emitir una alerta contra su uso y prohibirlo como una estrategia de salud pública.

\section{CONCLUSIÓN}

El CE es un dispositivo para administración de nicotina que genera adicción y efectos adversos al consumidor directo y a los expuestos al aire contaminado. Los reportes más recientes muestran daño alveolar agudo que lleva a hospitalización, ventilación mecánica y hasta muerte. Su uso como dispositivo para dejar de fumar tampoco ha podido ser establecido fehacientemente.

Los grupos más vulnerables a los efectos deletéreos del CE son los niños, adolescentes y jóvenes, quienes rápidamente se hacen adictos a esta nueva forma de uso de nicotina. A la luz de la evidencia actual, de no tomar medidas prontas, la morbilidad y mortalidad asociada a su uso podría presentarse también en países de la región.

Contribución de los autores: RAA ha participado en la concepción y diseño del artículo, recolección de información bibliográfica, redacción del artículo y revisión crítica del artículo. JL y KT han participado en la recolección de información bibliográfica y redacción del artículo. Todos aprobaron la versión final del artículo.

Fuentes de financiamiento: Autofinanciado.

Conflictos de interés: Los autores declaran no tener conflictos de interés.
6. Jiménez Ruiz CA, Solano Reina S, De Granda Orive JI, Signes-Costa Minaya J, De Higes Martínez E, Riesco Miranda JA y col. El cigarrillo electrónico. Declaración oficial dela Sociedad Española de Neumología y Cirugía Torácica (SEPAR) sobrela eficacia, seguridad y regulación delos cigarrillos electrónicos. Arch Bronconeumol. 2014;50(8):362-367. doi: 10.1016/j.arbres.2014.02.006

7. Herzog B, Gerberi J, Scott A. Equity Research: Tobacco-Nielsen C-store Data-E-cig \$ Sales Decline Moderates [sede Web]. Wells Fargo Securities: 2014 [acceso el 26 de marzo de 2019]. Disponible en: https://studylib.net/doc/13604808/equity-research-tobacco-nielsen-c-store-data--e-cig-\% 24 -sa...

8. Herzog B, Gerberi J, Scott A. Equity Research, "Tobacco Talk”-Q4 U.S Vapor Retailer Survey [sede Web]. Wells Fargo Securities: 2015 [acceso el 26 de marzo del 2019]. Disponible en: http:// www.ecigarette-politics. com/files/4q14-wells-fargo.pdf

9. Jamal A, Phillips E, Gentzke AS, Homa DM, Babb SD, King BA, et al. Current cigarette smoking among adults - United States, 2016. MMWR Morb Mortal Wkly Rep. 2018;67(2):53-59. doi:10.15585/mmwr.mm6702al 
10. Yoong SL, Stockings E, Chai LK, Tzelepis F, Wiggers J, Oldmeadow C, et al. Prevalence of electronic nicotine delivery systems (ENDS) use among youth globally: a systematic review and meta-analysis of country level data. Aust N Z J Public Health. 2018;42(3):303-308. doi:10.1111/17536405.12777

11. Maldonado V, Cruz JE. Reporte estadístico sobre consumo de drogas en el Perú y acciones en prevención y tratamiento - 2015 [Internet]. Lima: Comisión nacional para el desarrollo y vida sin drogas-DEVIDA; 2016 [acceso el 18 de diciembre del 2018]. Disponible en: http://www. simdev.gob.pe/2015/12/reporte-estadistico-sobre-consumo-de-drogas-en-el-peru-2015/

12. Oficina de las Naciones Unidas contra la Droga y el Delito. III Estudio epidemiológico andino sobre consumo de drogas en la población universitaria. Informe Regional, 2016. Lima: Oficina de las Naciones Unidas contra la Droga y el Delito; 2017 [acceso el 18 de noviembre del 2019]. Disponible en: https://www.unodc.org/documents/peruandecuador// Informes/Otros/Informe_Universitario_Regional.pdf

13. Cheng T. Chemical evaluation of electronic cigarettes. Tob Control. 2014;23:ii11-ii17.DOI:10.1136/tobaccocontrol-2013-051482

14. Trehy ML, Ye W, Hadwiger ME, Moore TW, Allgire JF, WoodruffJT, et al. Analysis of electronic cigarette cartridges, refill solutions, and smoke for nicotine and nicotine related impurities. J Liq Chromatogr Relat Technol. 2011;34(14):1442-1458. doi: 10.1080/10826076.2011.572213

15. Zhu SH, Sun JY, Bonnevie E, Cummins SE, Gamst A, Yin L, et al. Four hundred and sixty brands of e-cigarettes and counting: implications for product regulation. Tob Control. 2014;23:iii3-iii9. doi:10.1136/tobaccocontrol-2014-051670

16. Protano C, Avino P, Manigrasso M, Vivaldi V, Perna F, Valeriani F, et al. Environmental Electronic Vape Exposure from Four Different Generations of Electronic Cigarettes: Airborne Particulate Matter Levels. Int J Environ Res Public Health. 2018;15(10):2172. doi:10.3390/ijerph15102172

17. Huang J, Duan Z, Kwok J, Binns S, Vera LE, Kim Y, et al. Vaping versus JUULing: how the extraordinary growth and marketing of JUUL transformed the US retail e-cigarette market. Tob Control. 2019;28(2):146-151. doi:10.1136/tobaccocontrol-2018-054382

18. Jackler RK, Ramamurthi D. Nicotine arms race: JUUL and the high-nicotine product market. Tob Control. 2019;28(6):623-628. doi:10.1136/ tobaccocontrol-2018-054796.

19. Burch SG, Gann LP, Olsen KM, Anderson PJ, Hiller FC, Erbland ML. Effect of $\mathrm{pH}$ on nicotine absorption and side effects of aerosolized nicotine. Journal of Aerosol Medicine: Deposition, Clearance, and Effects in the Lung. 1993;6(1):45-52. [Acceso el 18 de noviembre del 2019]. Disponible en: https://nebraska.pure.elsevier.com/en/publications/effect-of-ph-onnicotine-absorption-and-side-effects-produced-by-

20. Leach J, Shockley L. TPM aqueous extract $\mathrm{pH}$ and extractable nicotine studies of major cigarette brands from $\mathrm{B} \& \mathrm{~W}$ and some domestic competitive companies. (TDO website). June 13, 1969. [acceso el 18 de noviembre del 2019]. Disponible en: https://www.tobaccodocuments.org/

21. Allem JP, Dharmapuri L, Unger JB, Cruz TB. Characterizing JULL-related posts on twitter. Drug Alcohol Depend. 2018;190:1-5. doi:10.1016/j. drugalcdep.2018.05.018.

22. Goniewicz ML, Boykan R, Messina CR, Eliscu A, Tolentino J. High exposure to nicotine among adolescents who use Juul and other vape pod systems ('pods'). Tob Control. 2018;28(6):676-677. doi:10.1136/ tobaccocontrol-2018-054565

23. Goniewicz ML, Kuma T, Gawron M, Knysak J, Kosmider L. Nicotine levels in electronic cigarettes. Nicotine Tob Res. 2013;15(1):158-166. doi: $10.1093 /$ ntr/nts103

24. U.S. Department of Health and Human Services. E-cigarette use among youth and young adults. A report of the surgeon general. Atlanta: U.S. Department of Health and Human Services, Centers for Disease Control and Prevention, National Center for Chronic Disease Prevention and Health Promotion, Office on Smoking and Health; 2016 [acceso el 26 de marzo de 2019]. Disponible en: https://e-cigarettes.surgeongeneral.gov/ documents/2016_sgr_full_report_non-508.pdf
25. Vanderkam P, Boussageon R, Underner M, Langbourg N, Brabant $Y$, Binder $\mathrm{P}$, et al. Efficacy and security of electronic cigarette for tobacco harm reduction: Systematic review and meta-analysis. Presse Med. 2016;45(11):971-985. doi:10.1016/j.lpm.2016.05.026

26. Liu X, Lu W, Liao S, Deng Z, Zhang Z, Liu Y, et al. Efficiency and adverse events of electronic cigarettes: A systematic review and meta-analysis (PRISMA-compliant article). Medicine (Baltimore). 2018;97(19):e0324. doi:10.1097/MD.0000000000010324

27. Bowler RP, Hansel NN, Drummond MB, Make BJ, Huh J, Dunton G, et al. E cigarette use in older Americans from the COPDGene cohort [Resumen]. Am J Respir Crit Care Med. 2015;191:A3908. [acceso el 26 de marzo de 2019]. Disponible en: https://www.atsjournals.org/doi/ abs/10.1164/ajrccm-conference.2015.191.1_MeetingResumens.A3908

28. Allehebi RO, Khan MH, Stanbrook MB. Efficacy and safety of electronic cigarettes for smoking cessation: A systematic review [Resumen]. Am J Respir Crit Care Med. 2015;191:A3715. [acceso el 26 de marzo de 2019]. Disponible en: https://www.atsjournals.org/doi/abs/10.1164/ ajrccm-conference.2015.191.1_MeetingResumens.A3715

29. Kasza KA, Ambrose BK, Conway KP, Borek N, Taylor K, Goniewicz ML, et al. Tobacco-product use by adults and youths in the United States in 2013 and 2014. N Engl J Med. 2017;376(4):342-353. doi:10.1056/ NEJMsa1607538

30. Dockrell M, Morrison R, Bauld L, McNeill A. E-cigarettes: prevalence and attitudes in Great Britain. Nicotine Tob Res. 2013;15(10):17371744. doi:10.1093/ntr/ntt057

31. Mowry JB, Spyker DA, Brooks DE, Zimmerman A, Schauben JL. 2015 Annual Report of the American Association of Poison Control Centers' National Poison Data System (NPDS): 33rd Annual Report. Clin Toxicol (Phila). 2016;54(10):924-1109. doi:10.1080/15563650.2016.1245421

32. Robot Palaczbot fumando cigarrillos con fines de investigación. Asimo [web] Cracovia: 2018. [acceso el 13 de noviembre del 2018]. Disponible en: http://www.asimo.pl/modele/palaczbot.php

33. Czogala J, Goniewicz ML, Fidelus B, Zielinska-Danch W, Travers MJ, Sobczak A. Secondhand exposure to vapors from electronic cigarettes. Nicotine Tob Res. 2014;16(6):655-662. doi:10.1093/ntr/ntt203

34. McAuley TR, Hopke PK, Zhao J, Babalan S. Comparison of the effects of e-cigarette vapor and cigarette smoke on indoor air quality. Inhal Toxicol. 2012;24(12):850-857. DOI:10.3109/08958378.2012.724728

35. Lim HH, Shin HS. Measurement of aldehydes in replacement liquids of electronic cigarettes by headspace gas chromatography-mass spectrometry. Bull Korean Chem Soc. 2013;34(9):2691-2696. doi:10.5012/ bkcs.2013.34.9.2691

36. Dicpinigaitis PV, Lee Chang A, Dicpinigaitis AJ, Negassa A. Effect of e-cigarette use on cough reflex sensitivity. Chest. 2016;149(1):161-165. doi:10.1378/chest.15-0817

37. Vardavas CI, Anagnostopoulos N, Kougias M, Evangelopoulou V, Connolly GN, Behrakis PK. Short-term pulmonary effects of using an electronic cigarette: impact on respiratory flow resistance, impedance, and exhaled nitric oxide. Chest. 2012; 141(6):1400-1406. doi:10.1378/chest.11-2443

38. Wieslander G, Norbäck D, Lindgren T. Experimental exposure to propylene glycol mist in aviation emergency training: acute ocular and respiratory effects. Occup Environ Med. 2001;58(10):649-655. doi:10.1136/ oem.58.10.649

39. McConnell R, Barrington-Trimis JL, Wang K, Urman R, Hong H, Unger $\mathrm{J}$, et al. Electronic cigarette use and respiratory symptoms in adolescents. Am J Respir Crit Care Med. 2017;195(8):1043-1049. doi:10.1164/rccm.201604-0804OC

40. Schweitzer RJ, Wills TA, Tam E, Pagano I, Choi K. E-cigarette use and asthma in a multiethnic sample of adolescents. Prev Med. 2017;105:226231. doi:10.1016/j.ypmed.2017.09.023

41. Cho JH, Paik SY. Association between electronic cigarette use and asthma among high school students in South Korea. PLoS One. 2016;11(3):e0151022. doi:10.1371/journal.pone.0151022

42. Lange P, Çolak Y, Ingebrigtsen TS, Vestbo J, Marott JL. Long-term prognosis of asthma, chronic obstructive pulmonary disease, and asthma-chronic obstructive pulmonary disease overlap in the Copenhagen 
City Heart study: a prospective population-based analysis. Lancet Respir Med. 2016;4(6):454-462. doi:10.1016/S2213-2600(16)00098-9

43. Kumral TL, Saltürk Z, Yildirim G, Uyar Y, Berkiten G, Atar Y, et al. How does electronic cigarette smoking affect sinonasal symptoms and nasal mucociliary clearance? [Resumen]. B-ENT. 2016;12(1):17-21. [acceso el 26 de setiembre del 2019]. Disponible en: https://europepmc.org/ Resumen/med/27097389

44. Higham AJ, Rattray N, Dewhurst JA, Goodacre R, Singh D. The effect of electronic cigarette exposure on innate immune cells [Resumen]. Am J Respir Crit Care Med. 2015;191:A2716. [acceso el 26 de setiembre del 2019]. Disponible en: https://www.atsjournals.org/doi/abs/10.1164/ ajrccm-conference.2015.191.1_MeetingAbstracts.A2716

45. Fain MD, Raju S, Lin VY, Tang L, Fernandez CM, Mazur M, et al. Effect of E-cigarette on airway epithelial ion transport and implications for mucociliary clearance defense. [Resumen]. Am J Respir Crit Care Med. 2015;191:A3868. [acceso el 26 de setiembre del 2019]. Disponible en: https://www.atsjournals.org/doi/abs/10.1164/ajrccm-conference.2015.191.1_MeetingAbstracts.A3868

46. Rowell TR, Lee S, Tarran R. Select E-cigarette flavors alter calcium signalin, cell viability and proliferation in lung epithelia [Resumen]. Am J Respir Crit Care Med. 2015;191:A2896. [acceso el 26 de setiembre del 2019]. Disponible en: https://www.atsjournals.org/doi/abs/10.1164/ ajrccm-conference.2015.191.1_MeetingAbstracts.A2896

47. Sherwood CL, Boitano S. Airway epithelial cell exposure to distinct e-cigarette liquid flavorings reveals toxicity thresholds and activation of CFTR by the chocolate flavoring 2,5-dimethypyrazine. Respir Res. 2016;17(1):57. doi:10.1186/s12931-016-0369-9

48. Saint-Criq V, Gray MA. Role of CFTR in epithelial physiology. Cell Mol Life Sci. 2017;74(1):93-115. doi:10.1007/s00018-016-2391-y

49. Ghosh A, Coakley RC, Mascenik T, Rowell TR, Davis ES, Rogers K, et al. Chronic E-cigarette exposure alters the human bronchial epithelial proteome. Am J Respir Crit Care Med. 2018;198(1):67-76. doi:10.1164/ rccm.201710-2033OC

50. Salathe M, García-Arcos I, Geraghty P, Schmid N, Dabo AJ, Cummins $\mathrm{N}$, et al. Nicotine in electronic-cigarettes causes inflammation, airway hyperreactivity and lung tissue destruction. [Resumen]. Am J Respir Crit Care Med. 2015;191:A4030. [acceso el 26 de setiembre del 2019]. Disponible en: https://www.atsjournals.org/doi/abs/10.1164/ajrccm-conference.2015.191.1_MeetingAbstracts.A4030

51. Sussan T, Gajghate S, Thimmulappa RK, Ma J, Kim J-H, Sudini K, et al. Exposure to electronic cigarettes impairs pulmonary anti-bacterial and anti-viral defenses in a mouse model. [Resumen]. Am J Respir Crit Care Med. 2015;191:A3944. [acceso el 26 de setiembre del 2019]. Disponible en: https://www.atsjournals.org/doi/abs/10.1164/ajrccm-conference.2015.191.1_MeetingAbstracts.A3944

52. Wu Q, Jiang D, Minor M, Chu HW. Electronic cigarette liquid increases inflammation and virus infection in primary human airway epithelial cells. PLoS ONE. 2014;9:e108342. doi:10.1371/journal.pone.0108342

53. Miyashita L, Suri R, Dearing E, Mudway I, Dove RE, Neill DR, et al. E-cigarette vapour enhances pneumococcal adherence to airway epithelial cells. Eur Respir J. 2018;51(2): pii:1701592. doi:10.1183/13993003.01592-2017

54. Crotty Alexander LE, Enany S, Hwang H, Sladewski K, Nizet V. Electronic cigarette vapor (ECV) exposure decreases Staphylococcus aureus susceptibility to macrophage and neutrophil killing. [Resumen]. Am J Respir Crit Care Med. 2014;189:A6624. [acceso el 26 de setiembre del 2019]. Disponible en: https://www.atsjournals.org/doi/abs/10.1164/ ajrccm-conference.2014.189.1_MeetingAbstracts.A6624

55. Van Zyl-Smit RN, Binder A, Meldau R, Semple PL, Evans A, Smith $\mathrm{P}$, Bateman ED, Dheda K.Cigarette smoke impairs cytokine respon- ses and BCG containment in alveolar macrophages. Thorax. 2014 69(4):363-70. doi:10.1136/thoraxjnl-2013-204229

56. Welz C, Canis M, Schwenk-Zieger S, Becker S, Stucke V, Ihler F, et al. Cytotoxic and genotoxic effects of electronic cigarette liquids on human mucosal tissue cultures of the oropharynx. J Environ Pathol Toxicol Oncol. 2016;35(4):343-54. doi:10.1615/JEnvironPatholToxicolOncol.2016016652

57. Leslie LJ, Vasanthi Bathrinarayanan P, Jackson P, Mabiala Ma Muanda JA, Pallett R, Stillman CJP, Marshall LJ. A comparative study of electronic cigarette vapor extracts on airway-related cell lines in vitro. Inhal Toxicol. 2017;29(3):126-36. doi:10.1080/08958378.2017.1318193

58. Moses ES, Wang TW, Jackson GR, Drizik E, Perdomo C, Park SJ, et al. Molecular impact of electronic cigarette exposure on airway epithelium. [Resumen]. Am J Respir Crit Care Med. 2015;191:A4026. [acceso el 26 de setiembre del 2019]. Disponible en: https://www.atsjournals.org/doi/ abs/10.1164/airccm-conference.2015.191.1_MeetingAbstracts.A4026

59. Blagev DP, Harris D, Dunn AC, Guidry DW, Grissom CK, Lanspa MJ. Clinical presentation, treatment, and short-term outcomes of lung injury associated with e-cigarettes or vaping: a prospective observational cohort study. Lancet. 2019; piiS0140-6736(19)32679-0. doi:10.1016/ S0140-6736(19)32679-0.

60. McCauley L, Markin C, Hosmer D. An unexpected consequence of electronic cigarette use. Chest. 2012;141(4):1110-1113. doi:10.1378/ chest.11-1334.

61. Long JL, Devabhaktuni S, Hadique S, Jain P. Diffuse alveolar hemorrhage due to electronic cigarette use. Am J Respir Crit Care Med 193;2016:A1862. [acceso el 26 de setiembre del 2019]. Disponible en: https://www.atsjournals.org/doi/abs/10.1164/ajrccm-conference.2016.193.1_MeetingAbstracts.A1862

62. CDC: Centers for Disease Control and Prevention [sede Web]. Atlanta: Department of Health and Human Services; [actualizada el 14 de noviembre de 2019; acceso el 14 de noviembre de 2019]. Outbreak of lung injury associated with the use of e-cigarette, or vaping, products [4 pantallas]. Disponible en: https://www.cdc.gov/tobacco/basic_information/e-cigarettes/severe-lung-disease.html

63. Christiani DC. Vaping induced lung injury. N Engl J Med. 2019 sep 6. DOI:10.1056/NEJMe1912032.

64. Henry TS, Kligerman SJ, Raptis CA, Mann H, Sechrist JW, Kanne JP. Imaging findings of vaping-associated lung injury. ARJ Am J Roentgenol. 2019;8:1-8. doi:10.2214/AJR.19.22251

65. Butt YM, Smith ML, Tazelaar HD, Vaszar LT, Swanson KL, Cecchini $\mathrm{MJ}$, et al. Pathology of vaping-associated lung injury. N Engl J Med. 2019;381(18):1780-1781. doi:10.1056/NEJMc1913069.

66. Mukhopadhyay S, Mehrad M, Dammert P, Arrossi AV, Sarda R, Brenner DS, et al. Lung biopsy findings in severe pulmonary illness associated with e-cigarette use (vaping): a report of eight cases. Am J Clin Pathol. 2019;XX1-10. doi:10.1093/ajcp/aqz182

67. Institute for global tobacco control [sede Web]. Baltimore: Johns Hopkins Bloomberg School of Public Health; [actualizada el 2 de noviembre de 2018; acceso el 29 de marzo de 2019]. Country laws regulating e-cigarettes: A policy scan [4 pantallas]. Disponible en: https://www.globaltobaccocontrol.org/e-cigarette_policyscan

68. Kennedy RD, Awopegba A, De León E, Cohen JE. Global approaches to regulating electronic cigarettes. Tob Control. 2017;26(4):440-445. doi:10.1136/tobaccocontrol-2016-053179

69. Infobae [sede Web]. Buenos Aires: TXH Medios S.A.; c2002-2019. Confirmaron la primera muerte por vapeo en México. [acceso 14 de noviembre 2019]. Disponible en: https://www.infobae.com/america/mexico/2019/11/09/confirmaron-la-primera-muerte-por-vapeo-en-mexico/ 